\title{
Research Paper \\ The Effectiveness of Acceptance and Commitment Therapy on Academic Procrastination in Medical Sciences Students of Zanjan University
}

\author{
Alireza Armani Kian ${ }^{1}\left(\mathbb{1}\right.$, Bahram Rostami², Seyyede Elnaz Moosavi ${ }^{3}$, Mahdi Maghbooli $^{4},{ }^{*}$ Ehsan Fakoor ${ }^{5}$
}

1. Department of Psychiatry, School of Medicine, Zanjan University of Medical Sciences, Zanjan, Iran.

2. Medical Education Development Center, Zanjan University of Medical Sciences, Zanjan, Iran.

3. Department of Clinical Psychology, School of Medicine, Zanjan University of Medical Sciences, Zanjan, Iran.

4. Department of Neurology, School of Medicine, Zanjan University of Medical Sciences, Zanjan, Iran.

5. Shahid Beheshti Medical Center, Zanjan University of Medical Sciences, Zanjan, Iran.

\begin{tabular}{|l|l}
$\begin{array}{c}\text { Use vour device to scan } \\
\text { and read the article online }\end{array}$ \\
ment Therapy (ACT) on Academic Procrastination in Medical Sciences Students of Zanjan University (Persian)] .Iranian Journal \\
of Psychiatry and Clinical Psychology. 2020; 26(2):142-153. http://dx.doi.org/10.32598/ijpcp.26.2.2817.1
\end{tabular}

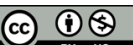

Received: 28 Nov 2019

Accepted: 22 May 2020

Available Online: $01 \mathrm{Jul} 2020$

Key words:

Commitment-based

therapy, Academic

procrastination,

Students

\section{A B STRACT}

Objectives Procrastination and academic failure are factors that are on the rise among medical universities and threaten the educational system. Therefore, it is necessary to identify interventions that can reduce these factors. The present study aimed to evaluate the effectiveness of Acceptance and Commitment Therapy (ACT) on academic procrastination in Medical Sciences students of Zanjan University, Zanjan City, Iran.

Methods This study was a quasi-experimental study with a pre-test \& post-test design and case and control groups. The statistical population was all students with academic failure who were referred to the University Counseling Office during the academic year 1996-7, out of whom 38 were enrolled in the study considering the inclusion criteria and academic procrastination score. The study data were collected using the standard Solomon's and Rothlobom questionnaire. Students' academic performance was also measured by their semester grade point average. Covariance analysis and analysis of covariance were used for data analysis in SPSS V. 24.

Results The mean academic procrastination was significantly different between the two groups at the post-test $(\mathrm{P}<0.05)$. Group $\mathrm{F}$ was statistically significant in academic procrastination equal to 257.553 $(P<0.05)$, so the effectiveness of ACT on academic procrastination and its components was confirmed. Concerning academic performance, the $F$ of the group factor was equal to 9.59 and at the level of $P<0.05$ which was statistically significant.

Conclusion The results show the effectiveness of ACT on student's procrastination and academic failure.

\section{Extended Abstract}

\section{Introduction}

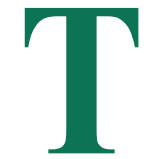

oday, one of the necessary measures to optimize education in a country is examining the effective factors on the academic growth and motivation for achievement in university students [1]. Academic procrastination is delaying the tasks and activities associated with studying. It is one of the significant factors related to students' academic performance and neglecting it could bring about irredeemable repercussions [2]. According to Molen, procrastination is frequent among students [3] as its prevalence ranges from at least

* Corresponding Author:

Ehsan Fakoor

Address: Shahid Beheshti Medical Center, Zanjan University of Medical Sciences, Zanjan, Iran.

Tel: +98 (912) 5414042

E-mail: ehsan.fakour@gmail.com 
$71 \%-91 \%$; its acute kind has been estimated to range from $23 \%-31 \%$ [4]. Academic procrastination is effective in preparing for exams, doing homework, and attending class sessions [5]. It influences mental health and academic achievement in university students and its most crucial outcome is academic underachievement [12].

Because of its cognitive nature, proper psychological interventions could presumably prevent academic procrastination and underachievement. There are several therapeutic interventions for academic procrastination, including cognitive behavioral therapy which has had the most application, and many studies have been devoted to it [13-16]. In this regard, some studies [20-22] considered the effectiveness of third-wave therapies; however, more studies are required to determine the clinical effectiveness of these therapies as they are new-found. We could point out Acceptance and Commitment Therapy (ACT) among the interventions.

Some mental factors such as stress, depression, and anxiety can also intensify academic underachievement and procrastination in university students. Studies have shown that ACT is effective in reducing stress, anxiety, depression, and cognitive dissonance. Thus it seems that this therapy could reduce academic procrastination and as a result underachievement in university students. Moreover, studies investigating the procrastination of students of Zanjan University of Medical Sciences showed that these factors have spread among the students [29-31]. Therefore, the purpose of this study is to determine the effectiveness of ACT in reducing the academic procrastination of students at Zanjan University of Medical Sciences.

\section{Methods}

The present interventional and quasi-experimental study was done with a pre-test-post-test design and a control group. All of the questionnaires were completed by the subjects in the pre-test phase. Furthermore, the study variables for the intervention group and the control group were calculated in both pre-test and post-test to determine and compare the effectiveness of the intervention. The study population included all 90 students reported with academic underachievement to the Zanjan University of Medical Sciences counseling office in the 2017-2018 academic year. Thirty-eight students were included in the study in two groups of 19 members by the purposive method. The researcher and data coding method was used in all stages of the study to prevent sample pollution. There were no sample losses in both groups after the final selection. The inclusion criteria were a GPA of less than 12 in the semester (conditional pass), a higher grade than the cut-off point allotted in the procrastination questionnaire and exclusion criteria consisted of psychotic disorders, personality disorders, depression, anxiety, and structured clinical interview based on SCID I (Structured Clinical Interview for DSM-IV Axis I Disorders) to assure the absence of other problems such as depression in subjects in the first stages of screening.

\section{Results}

Thirty-eight students with high academic procrastination participated in this study. Female students were most frequent in both groups. There were 14 and 15 female students in the intervention group and control group, respectively. The most frequent age range was 18-21 years and the most frequent participating subjects were medical students in both groups. Moreover, the F coefficient related to the group factor in academic procrastination was 257.553 and statistically significant $(\mathrm{P}<0.05)$ level in post-test and MANCOVA test. Therefore, the hypothesis of the effectiveness of ACT on procrastination and its components was confirmed. Besides, the significance of the F effect of the pre-test showed that the sensitization of the pre-test had a decreasing or increasing effect on the experimental intervention (Table 1).

\section{Discussion}

Academic procrastination and underachievement include different aspects, one of the important and significant of

Table 1. The influence of ACT on academic procrastination of university students and its components

\begin{tabular}{cccccc}
\hline Dependent Variables & Sum of Squares & df & Mean of Squares & F Coefficient & Sig. \\
\hline $\begin{array}{c}\text { Academic procrastination } \\
\text { (overall) }\end{array}$ & 4647.108 & 1 & 4647.108 & 257.553 & 0.001 \\
$\begin{array}{c}\text { Procrastination of preparing } \\
\text { for exams }\end{array}$ & 341.894 & 1 & 341.894 & 55.807 & 0.001 \\
\hline $\begin{array}{c}\text { Procrastination of doing } \\
\text { homework }\end{array}$ & 693.891 & 1 & 693.891 & 120.080 & 0.001 \\
$\begin{array}{c}\text { Procrastination of term } \\
\text { papers and seminars }\end{array}$ & 544.640 & 1 & 544.640 & 85.236 & 0.001 \\
\hline
\end{tabular}


which is psychological factors. Accordingly, the study of such factors needs to be multi-dimensional and comprehensive. In addition to education, cultural and student domains and counseling have active roles in solving the problems of students with academic underachievement. This process indicates the significance of psychological factors in the underachievement of university students. Constant and accurate use of psychological approaches should be incorporated in solving these issues. The results of this study confirmed this conclusion. The results showed the favorable effects of psychotherapies, particularly ACT. Furthermore, given the prevalence of procrastination and underachievement among university students, the results of this study could be effective in reducing the factors.

\section{Ethical Considerations}

Compliance with ethical guidelines

This study is confirmed ethically by the registration ID IR.ZUMS.REC.1937.394. All ethical principles were observed in this study. The participants had the right to withdraw from the research at their will. Also, all of the information associated with participants were kept confidential during the research process.

Funding

This research was funded by the National Agency for Strategic Research in Medical Education, Tehran, Iran with a registration number of 961073 .

Authors contributions

Conceptualization: Ehsan Fakour and Alireza Armani Kian; Research: Bahram Rostami, Mehdi Maqbooli, Ehsan Fakour; Editing and finalizing: Seyedeh Elnaz Mousavi.

\section{Conflicts of interest}

The authors declared no conflicts of interest.

\section{Acknowledgements}

The authors of this article would like to thank the management and colleagues at the National Agency for Strategic Research in Medical Education and all of the participating students in this research. 


\section{اثربخشى درمان مبتنى بر تعهد و يذيرش بر اهمال كارى تحصيلى دانشجويان دانشكاه علوميزشكى

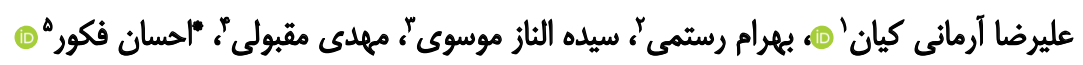

1. بروه روانيزشكى، دانشكده يزشكى، دائشكاه علوميزشكى زنجان، زنجان، ايران.

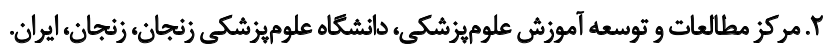

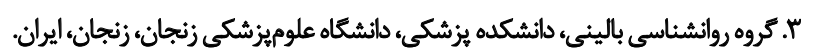

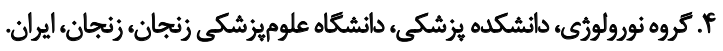

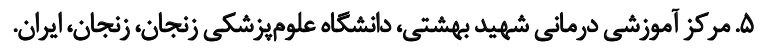

\section{حكيد}

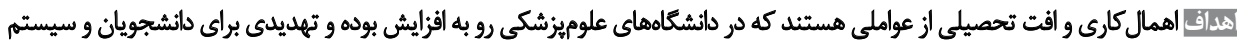

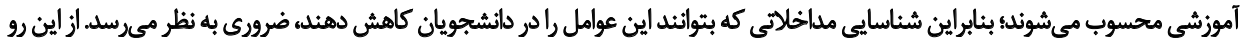

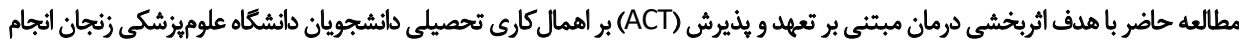

كرفت.

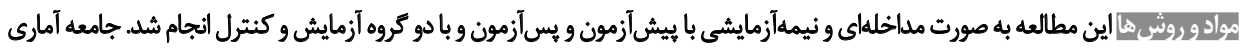

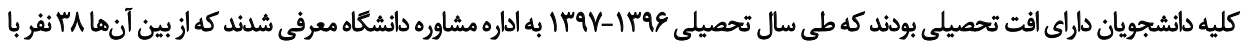

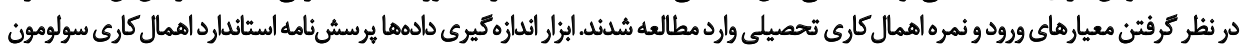

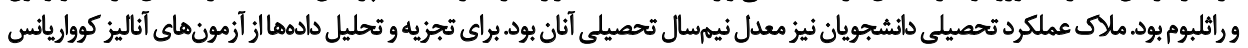

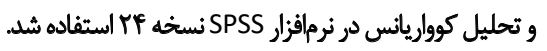

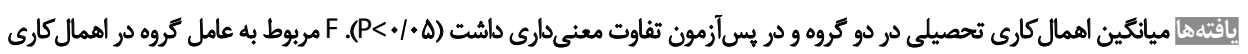

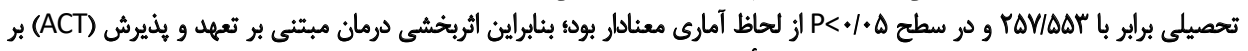

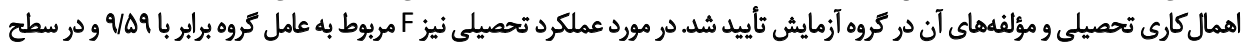

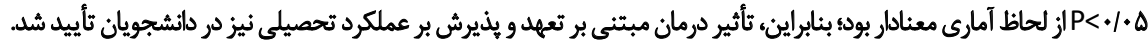

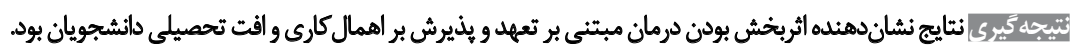

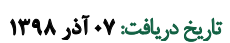

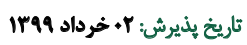

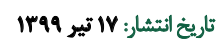

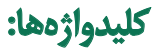
درمان مبتنى بر تعهد

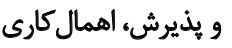
تحصيلى، دانشجويان

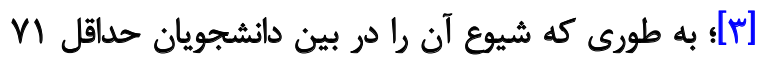

مقدمه

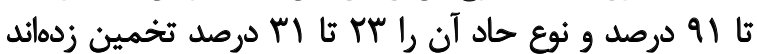

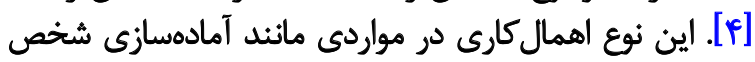
براى امتحان، انجام تكاليف و حضور در در جلمارد مانسات و انجام تكاليف

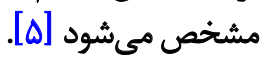
دورا معتقد است كه به تأخير انداختن و يا اجتناب از انجام

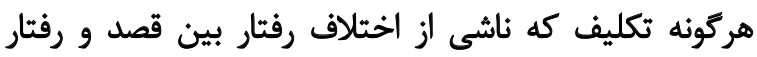

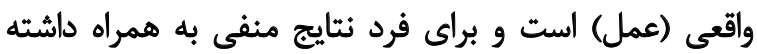

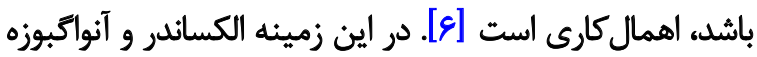

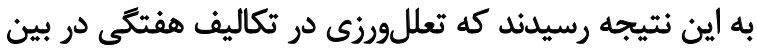

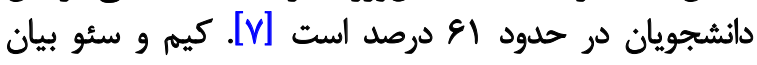

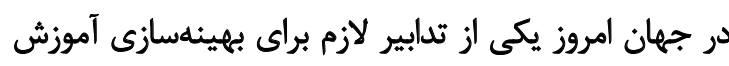

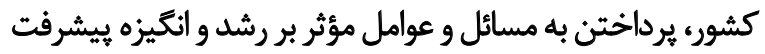

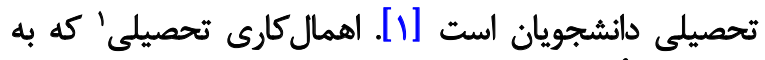

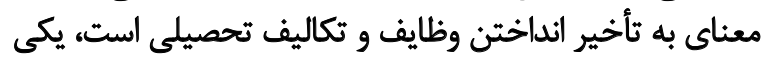

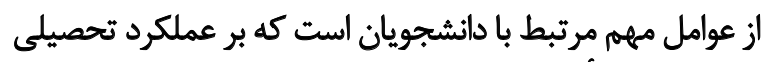

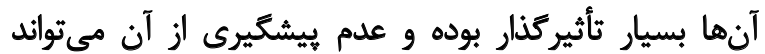

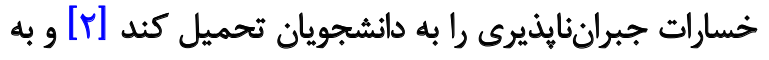

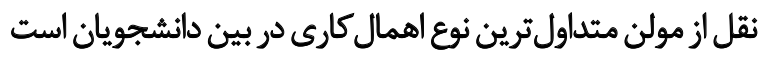

1. Academic procrastination

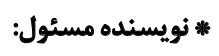

احسان فكور

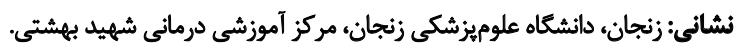
تلفئ: نو ehsan.fakour@gmail.com يست الكترونيكي: تئي 
روانشناختى است. مراد از انعطافيذيرى روانشناختى، توانايى

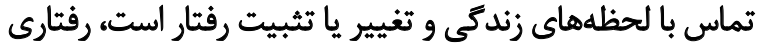

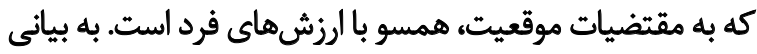

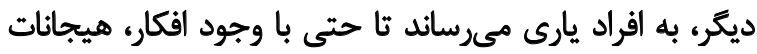

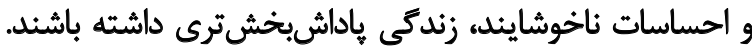

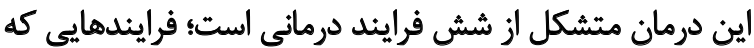

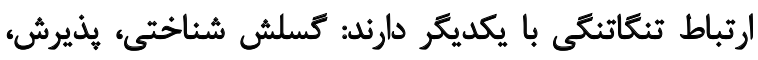

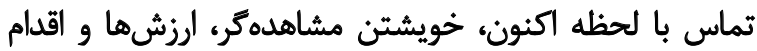

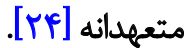

مطالعات انجام كرفته در زمينه اثربخشى درمان مبتنى بر تعهيد و

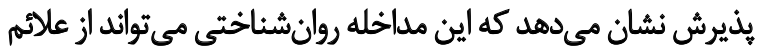

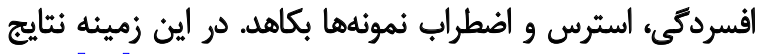

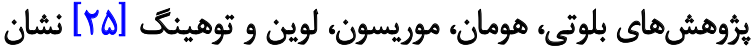

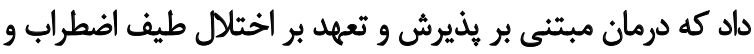

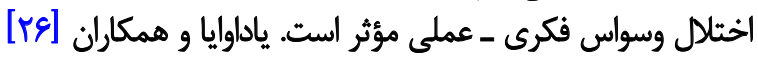

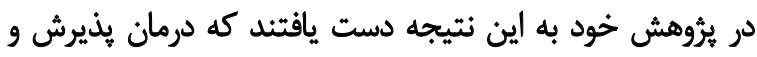

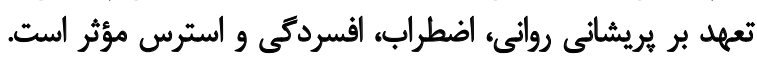

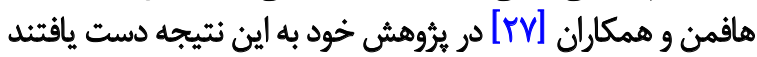

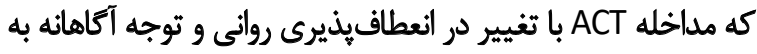

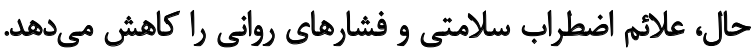

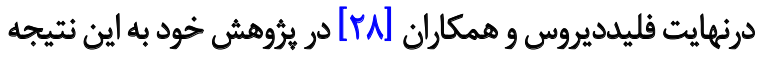
دست يافتند كه انعطافيذيرى در طول مداخله موجب كاهش افسردمى و اضطراب مىشود.

نتايج مطالعات در زمينها فت تحصيلى واهمال كارى تحصيلى نشان

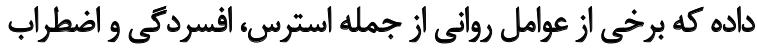

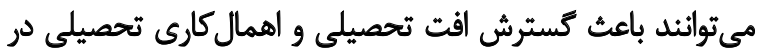

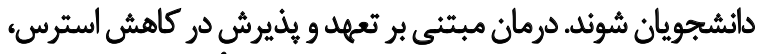

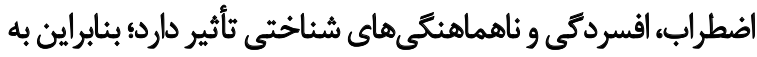

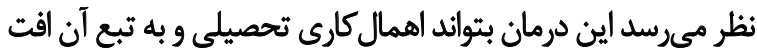

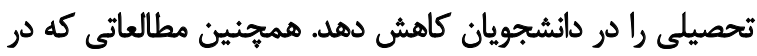

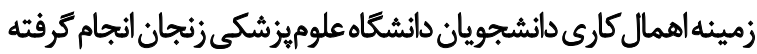

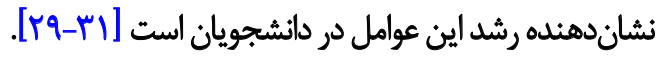

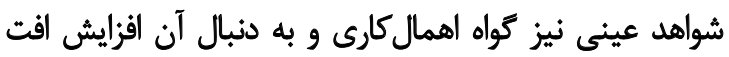

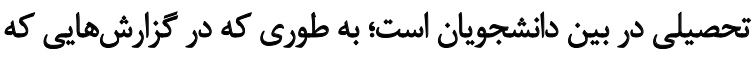

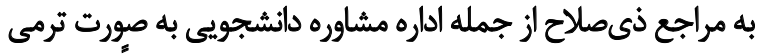

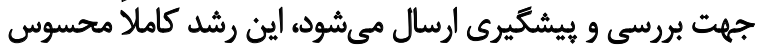

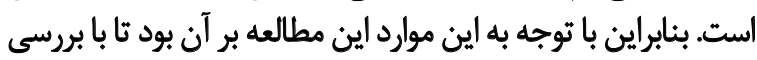

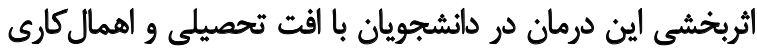

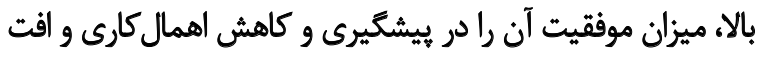

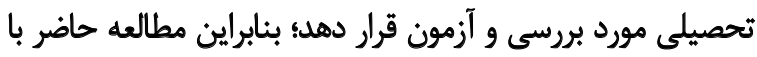

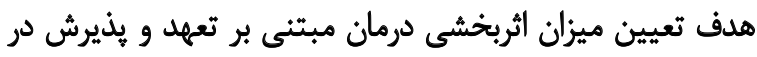

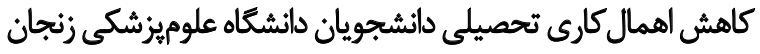

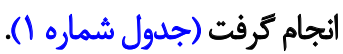

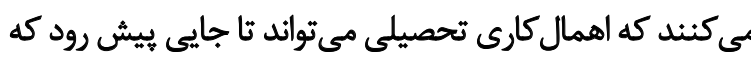

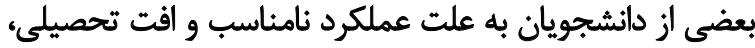

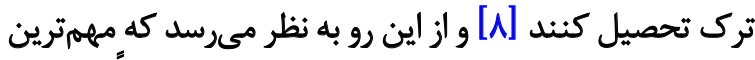

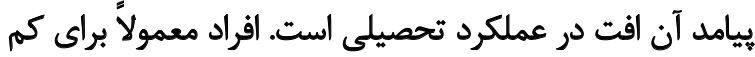

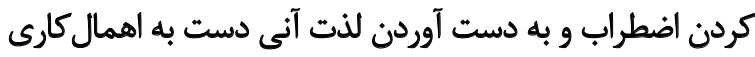

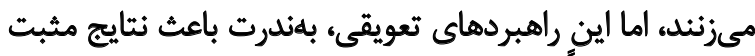

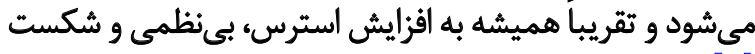

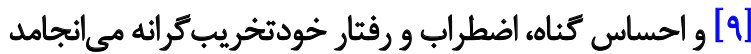

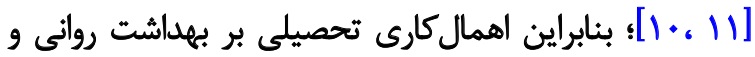

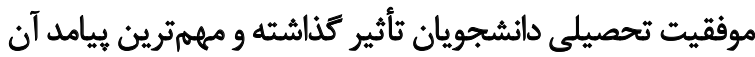
افت تحصيلى است [1/T]

در اين بين كمان مىرود با توجه ماهيت شناختى اينَّونه

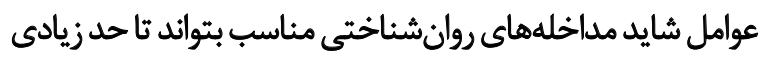

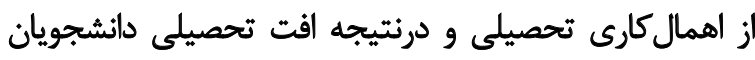

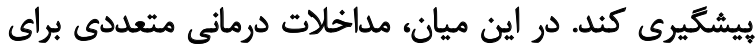

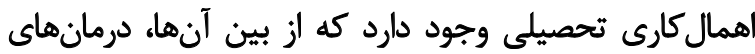

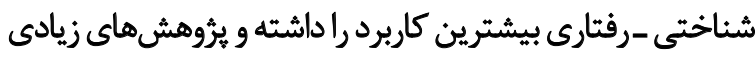

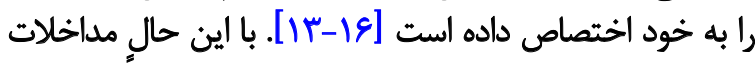

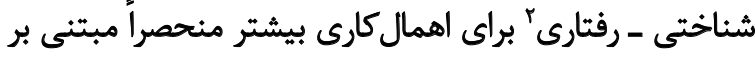

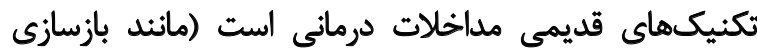

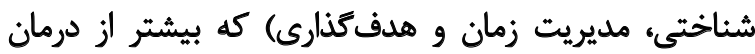

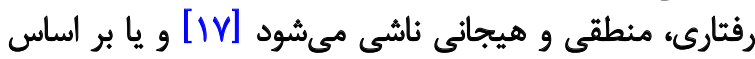

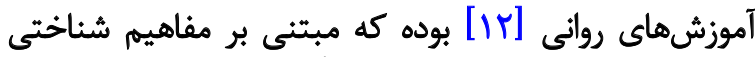

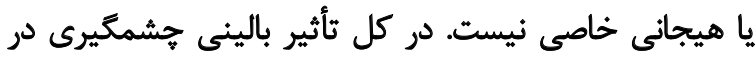

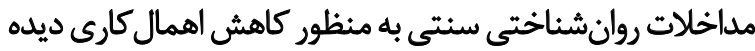

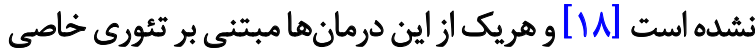

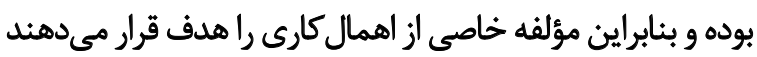

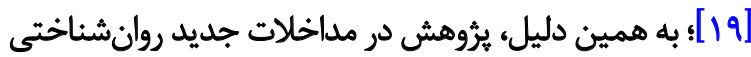

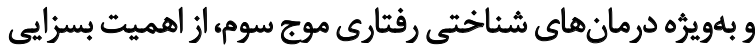

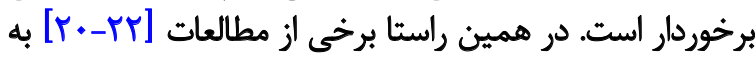

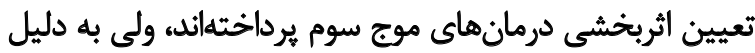

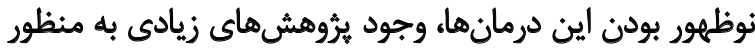

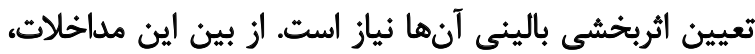

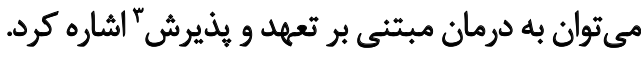
ريشه در رفتاركرايى دارد، اما به وسيله فرايندهاى

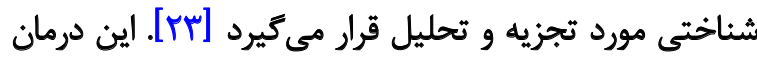

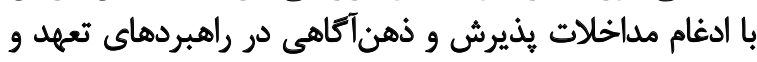

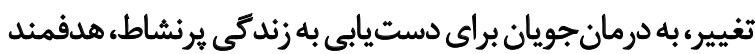

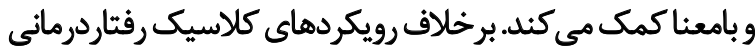

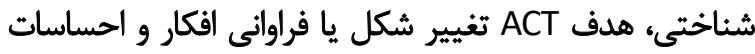

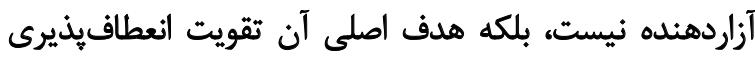

2. Cognitive-behavioral therapy

3. Acceptance and Commitment Therapy (ACT) 
اهمال كاري،تمايل براى شركت در ثرؤهش و عدم مصرف الكل

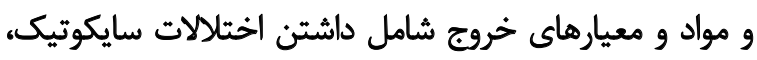

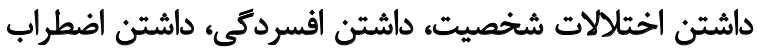

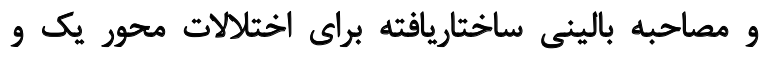

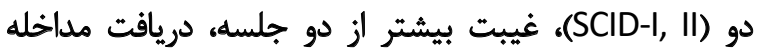

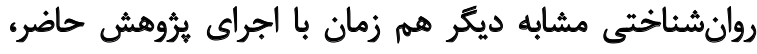

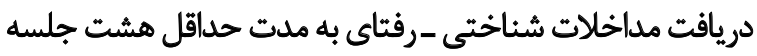

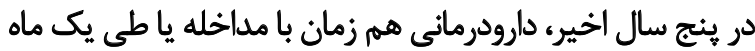

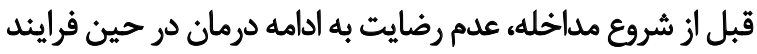

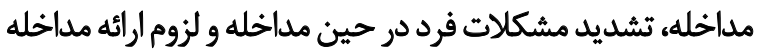

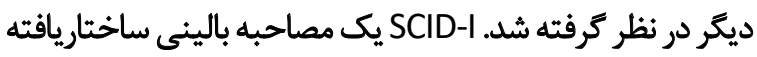

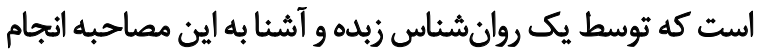

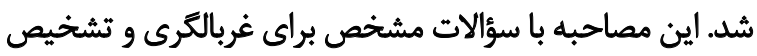

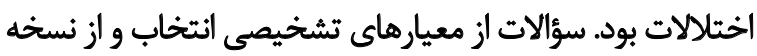

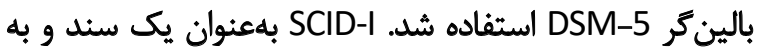

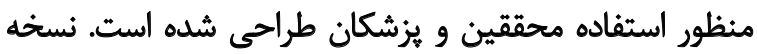

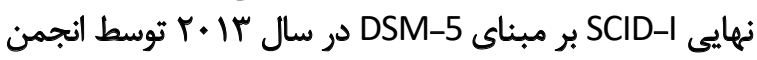

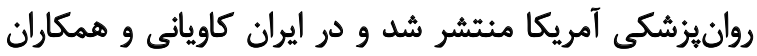
نسخه انغليسى اين ابزار را به فارسى ترجما مرئه كردهاند.

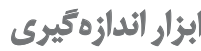

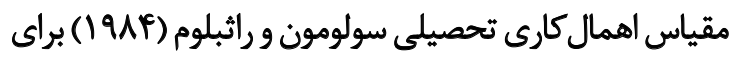

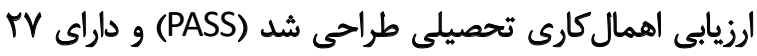

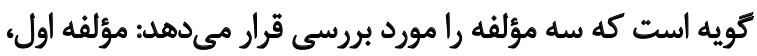

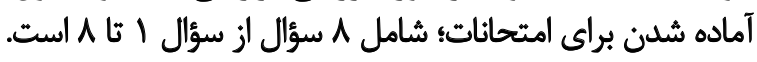

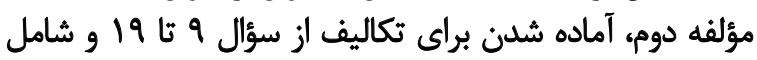

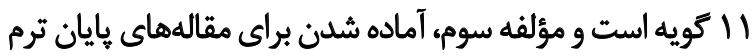

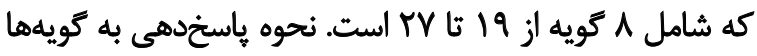

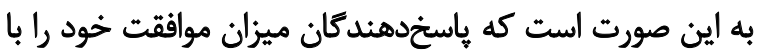

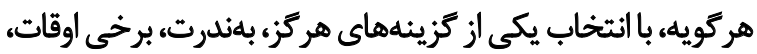

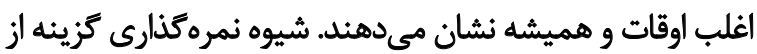

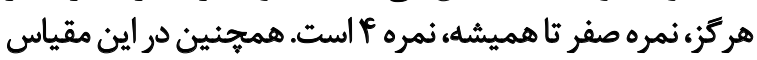

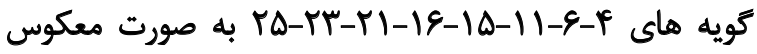

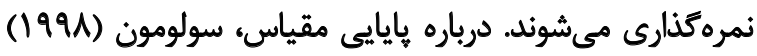

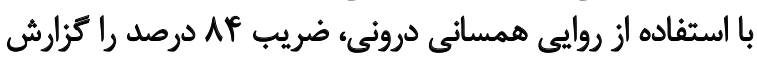

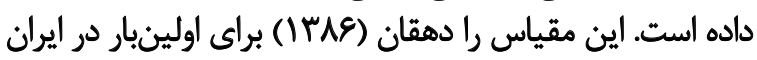

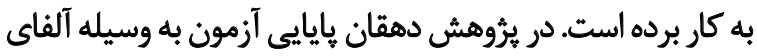

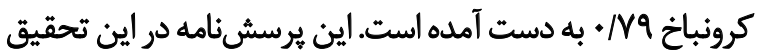

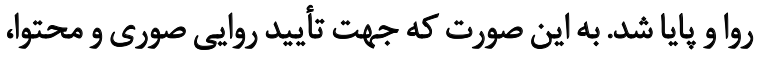

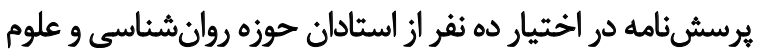

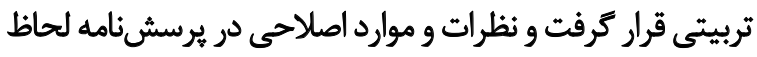

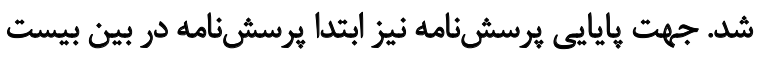

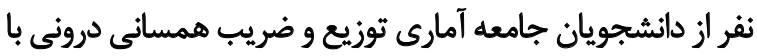

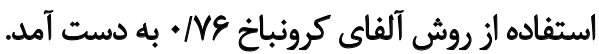

اين مطالعه به صورت مداخلهاى و نيمهآزمايشى با باز

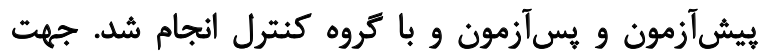

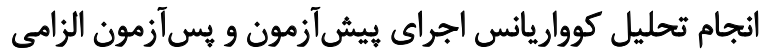

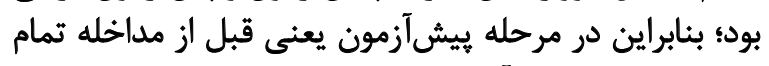

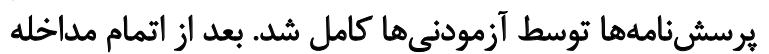

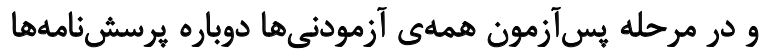

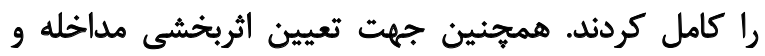

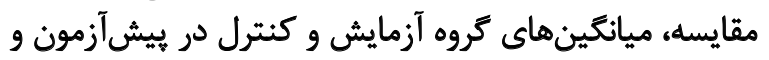

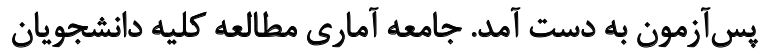

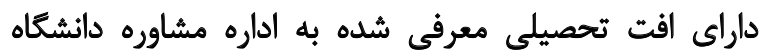

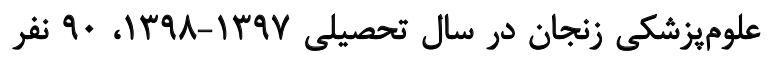

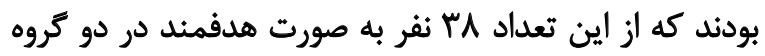

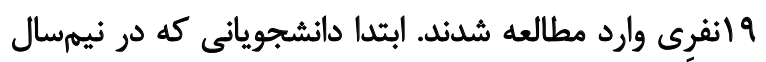

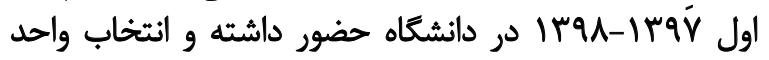

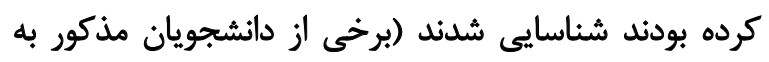

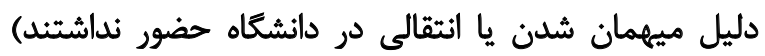

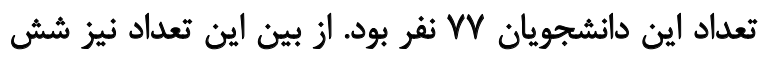

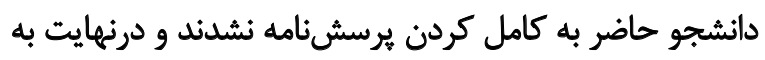

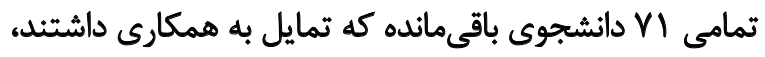

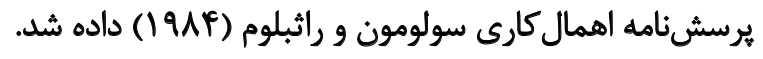
يس از تكميل يرسشئامه در اين مرحله نمرات اهمال كارى

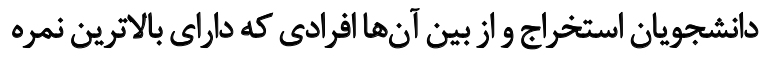

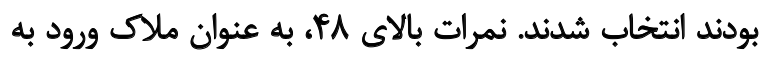

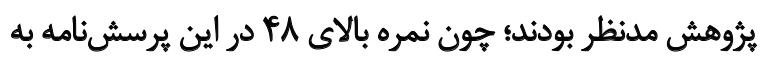

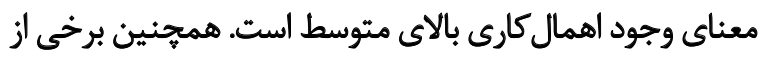

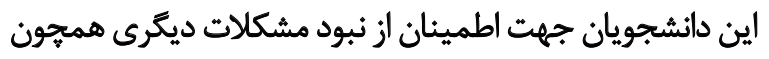

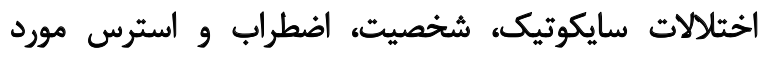

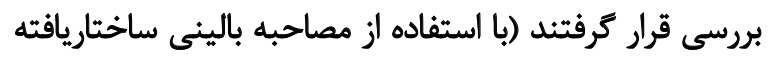

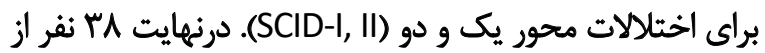

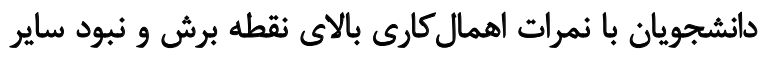

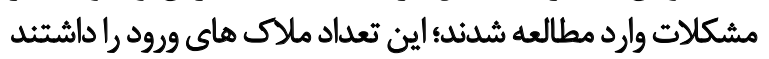

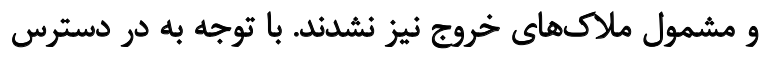

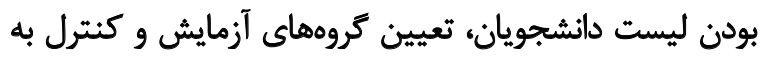

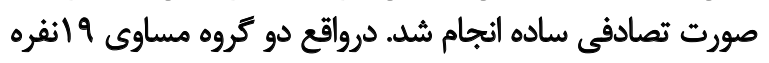

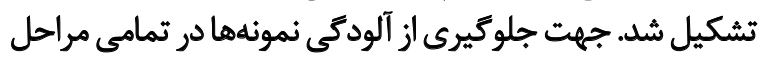

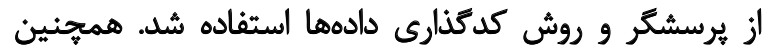

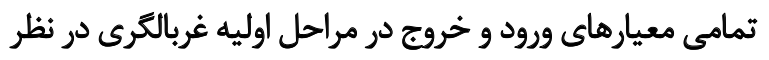

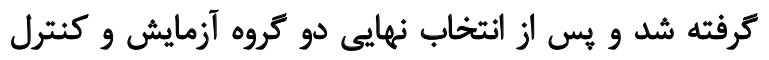

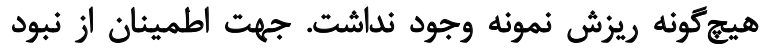

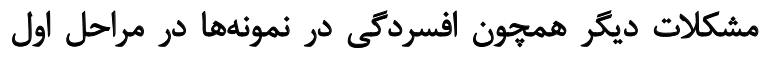

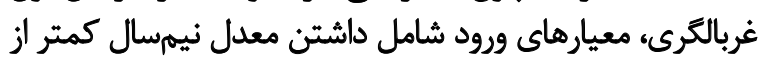

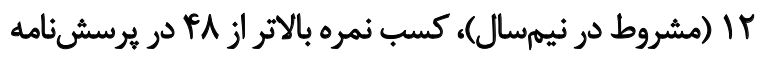




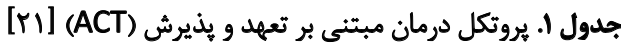

\begin{tabular}{|c|c|c|}
\hline مداخله & جلسه & مائولها \\
\hline آشنايي دائشجويان با دوره و ساير افراد كروهو وتوزيع بروشورهاى اطلاعرسانى & 1 & معارفه دوره و شركت كندكان \\
\hline برقرارى رابطه درمانى، صحبت در مورد درمان و اهداف آن & r & مازٔول 1. مقدهه و دستور جلسه درمان \\
\hline 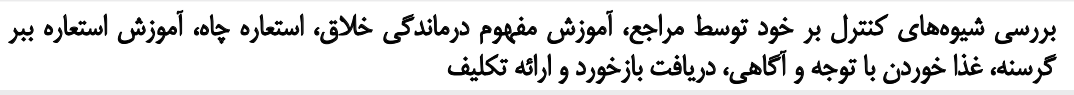 & $r$ & مائول Y. تغيير رفثار و ذذهن آكاهيى \\
\hline 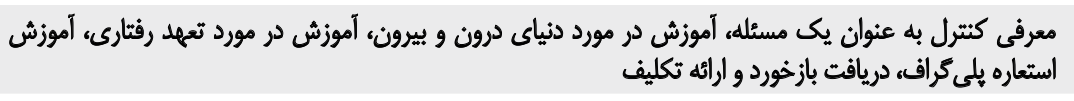 & $r$ & مازّول س. ارزش ها \\
\hline 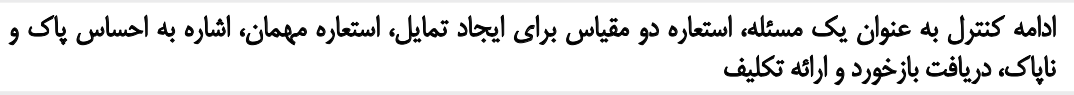 & $\Delta$ & مارٔول f. شفافسازى ارزش ها و اهداف \\
\hline بازخورد و ارائه تكليف، أموز استعاره اعداد و اتوبوس، بررسى افكار ناخوشايند به عنوان واقعيت، تعهات رفتارى، دريافت & $\varepsilon$ & مازول هـ كستشكى \\
\hline خريافت بازخورد و ارئائه تكليف (صحبت از من و من مشاهدهر)، بررسى ارزشها، بررسى فرم شحح ارزشها، تمرين ارزشها، & $v$ & مارّول \& عمل متعهدانه \\
\hline 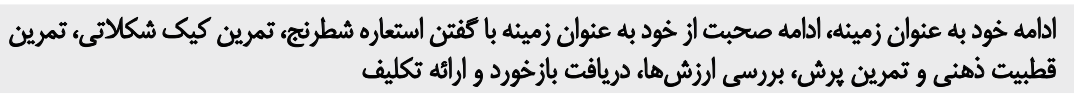 & $\wedge$ & مارٔول V. عمل متعهبائه \\
\hline ارزيابى تعهد به عمل، استعاره حباب و نهال، أموزش تمرين محتوا روى كارت، ارزيابى ثعهد به عمل & $q$ & مارُول م رضايت \\
\hline جمعبندى جلسات درمانى & 1. & جمعبندى جلسات \\
\hline
\end{tabular}

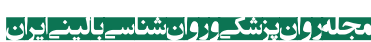

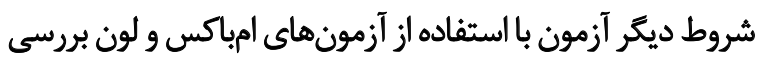

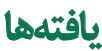

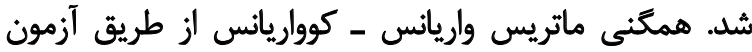

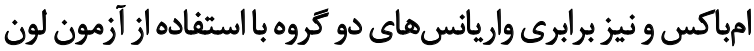

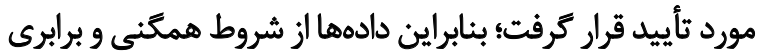

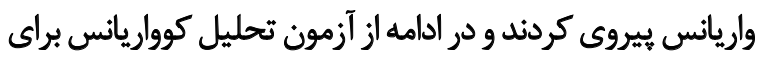
بررسى اثربخشى مداخله استفاده شد.

همان كونه كه در جدول شماره r مشاهده مي مئود، اثرات

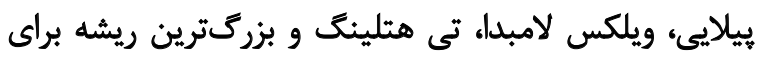

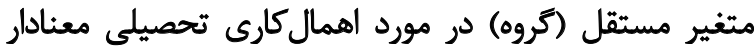

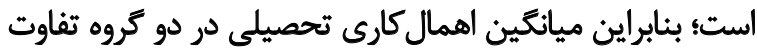

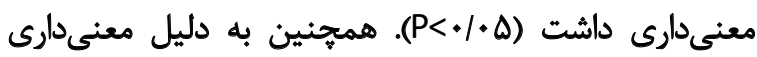
شاخص F جندمثغيرى، تحليل اثرات بين آزمودنى لازم بود.

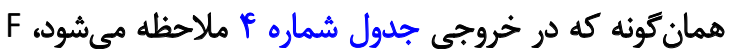

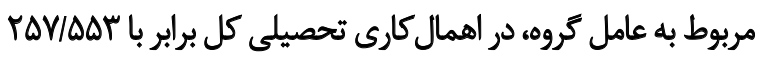

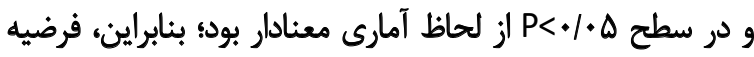

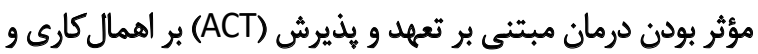

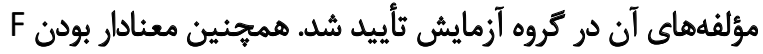

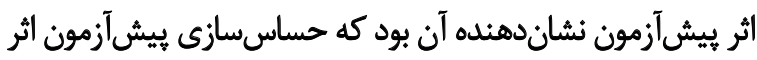
كاهشى يا افزايشى بر مداخله آزمايشى برجا برانداشته است.

بحث

تحقيق حاضر با هدف تعيين اثربخشى درمان مبتنى بر تعهد و يذيرش (ACT) بر اهمال كارى تحصيلى دانشجويان دانشكاه دان مدان

در مطالعه حاضر ^ץ نفر از دانشجويان داراي افت تحصيلى كه

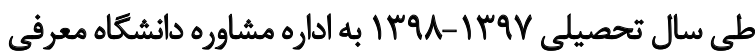

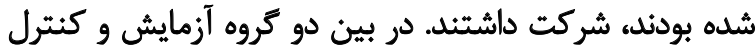

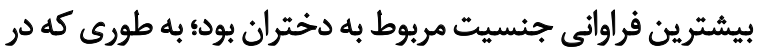

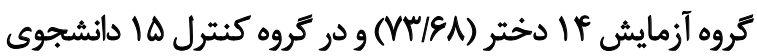

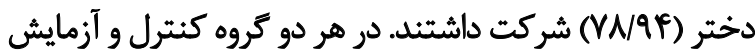

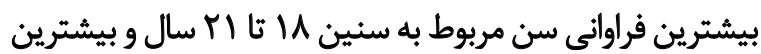

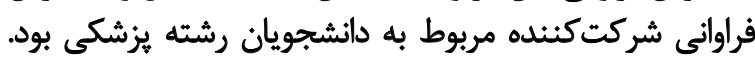

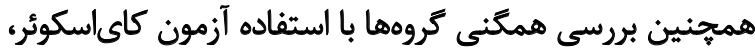

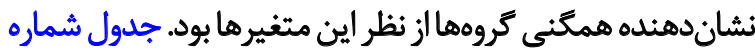

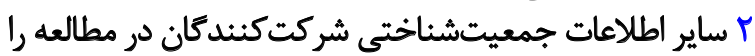
نمايش مى دهد.

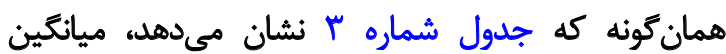

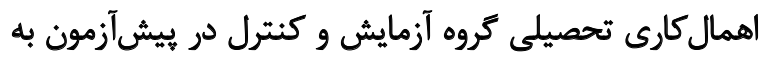

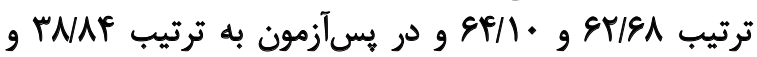
ترتيب أF/AF

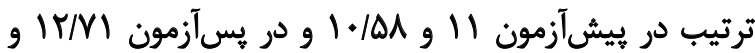

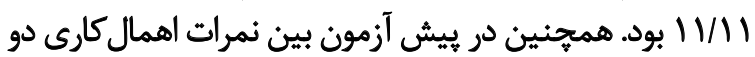

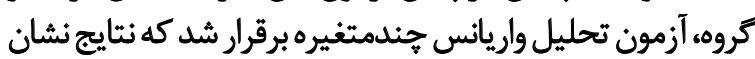

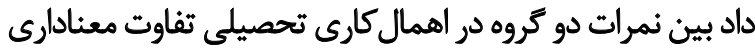

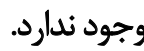

جهت انجام تحليل كوواريانس با توجه به نرمال بودن دادهها، 
جدول r. توزيع فراوانى دانشجويان برحسب متغيرهاي جمعيتشناختى مطالعه

\begin{tabular}{|c|c|c|c|c|c|}
\hline \multirow{2}{*}{\multicolumn{2}{|c|}{ مجموع }} & \multicolumn{2}{|c|}{ تعداد (درصد) } & \multirow{2}{*}{\multicolumn{2}{|c|}{ متغيرهاى جمعيتشناختى مطالعه }} \\
\hline & & كتترل & آزمايش & & \\
\hline \multirow{4}{*}{$\begin{array}{l}X^{2}=1.155 \\
P=0.283\end{array}$} & หq (Ne) & $10(\mathrm{NA})$ & If $\left(n^{\prime}\right)$ & دختر & \multirow{4}{*}{ جنسيت / فراوانى (درصل) } \\
\hline & 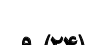 & & & & \\
\hline & I(IT) & F(IT) & $\Delta(T V)$ & 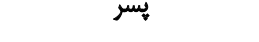 & \\
\hline & ra & 19 & 19 & مجموع & \\
\hline \multirow{9}{*}{$\begin{array}{l}X^{2}=6.10 \\
P=0.191\end{array}$} & $\mathbb{I r}(r \Delta)$ & $\varepsilon(M)$ & $V(r V)$ & يزشكى & \multirow{9}{*}{ رقتئه تحصيلي / فراواتي } \\
\hline & $11($ (T) & $\Delta(r \varepsilon)$ & $\&(M T)$ & داروسازى & \\
\hline & $r(\Lambda)$ & $r(1 \cdot)$ & $1(\Delta)$ & دندانيز شكىى & \\
\hline & $r(\Lambda)$ & $r(1 \cdot)$ & $1(\Delta)$ & 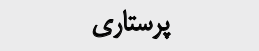 & \\
\hline & $r(\Delta)$ & $1(\Delta)$ & $1(\Delta)$ & 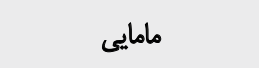 & \\
\hline & $r(\Delta)$ & $1(\Delta)$ & $1(\Delta)$ & علوم آزمايشكاهي & \\
\hline & $r(\Delta)$ & $1(\Delta)$ & $1(\Delta)$ & همهندسي بهلداشت حرفهاي & \\
\hline & $r(\Delta)$ & $1(\Delta)$ & $1(\Delta)$ & هوشبرى & \\
\hline & ra & 19 & 19 & مجميع & \\
\hline \multirow{5}{*}{$\begin{array}{l}X^{2}=3.278 \\
P=0.351\end{array}$} & $A(r))$ & $f(r)$ & $F(r))$ & 9 & \multirow{5}{*}{ سال ورود به داتشكاه / } \\
\hline & $1 \cdot(r \varepsilon)$ & $a(r)$ & $\Delta(Y \varphi)$ & $9 f$ & \\
\hline & $\mathbb{1 r}(r \Delta)$ & $v(r v)$ & $8(M T)$ & $9 \Delta$ & \\
\hline & $V(M)$ & $r(19)$ & $F(r)$ & $\vartheta$ & \\
\hline & ra & 19 & 19 & مجموع & \\
\hline
\end{tabular}

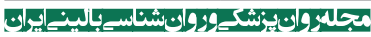

نيز يُوهشكر كاربرد درمانهاى نسل سوم شناختىرفتارى از

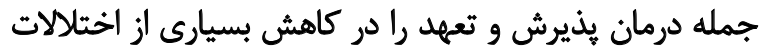

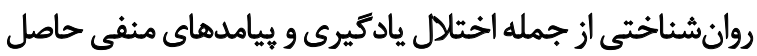

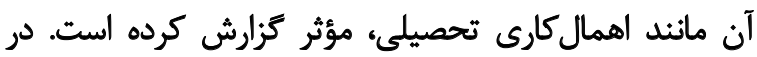

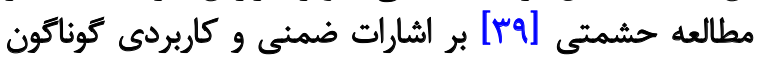

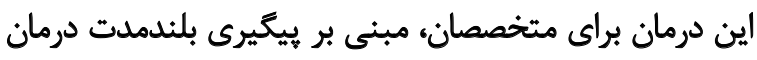

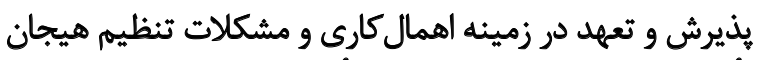

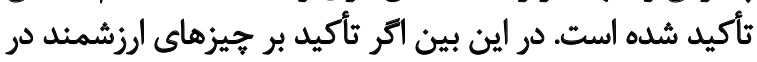

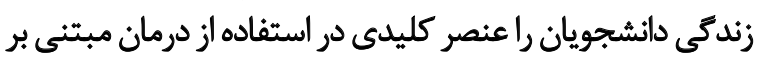

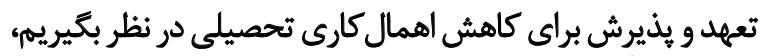

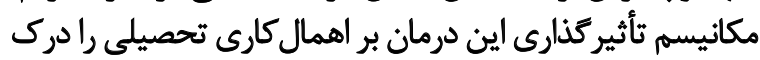

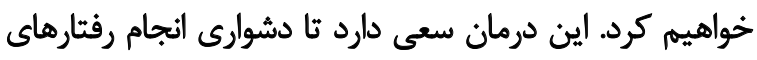

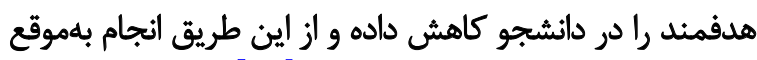

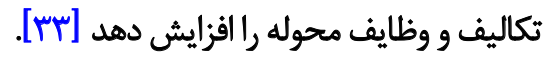

در واقع اهمال كارى تحصيلى به عنوان يك ابزار براى مقابله با استرس و اضطراب ناشى از انجام يك كار يا يا تكليف توسط ئران
علوميزشكى زنجان انجام كرفت. نتايج بررسى فرضيه انهاي

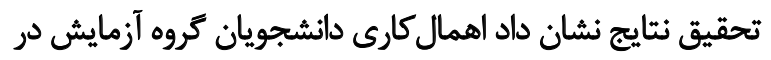

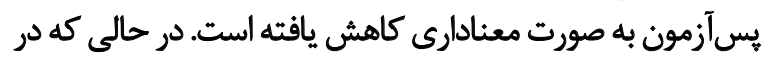

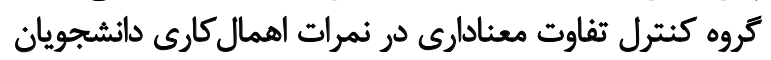

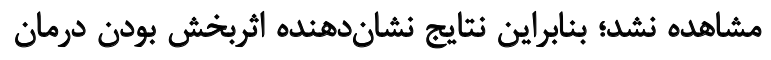

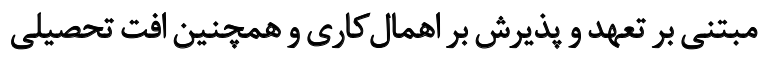

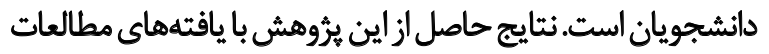

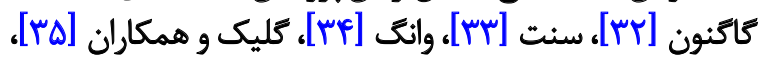

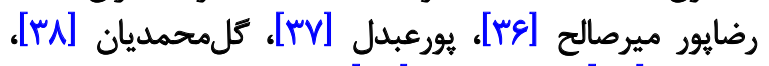

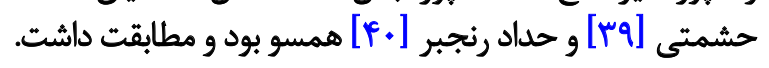

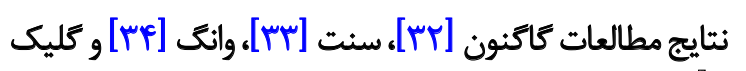

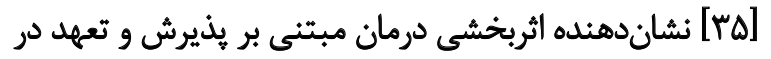

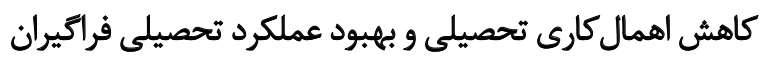

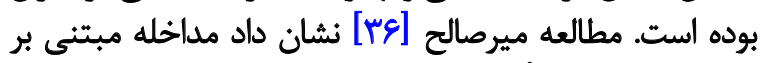

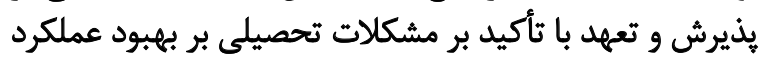

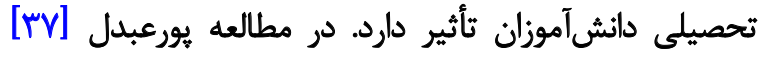


جدول ץ. نتايج آزمونهاى معنى دارى تحليل كوواريانس جندمتغيره

\begin{tabular}{|c|c|c|c|c|c|c|c|}
\hline اندازه اثر & $\mathbf{P}$ & درجه آزادى خطا & درجه أزادى اثر & $\mathbf{F}$ & ارزش & آزمون & متغير وابسته \\
\hline . / 194 & $.1 . .1$ & $r 1 / .$. & $r / \ldots$ & ASIVA & ./199 & اثر ييلايع & \\
\hline ./A9Y & $.1 . .1$ & $r / \ldots$ & $r / \ldots$ & NSIVA & $\cdot N \cdot v$ & لامبداي ويلكز & اهمال كارى \\
\hline / /A9T & $.1 . .1$ & $r / \ldots$ & $r / .$. & AS/VD & Nire. & اثر هثلينك & تحصيلى \\
\hline / /199 & $+1+\infty$ & $r / . .$. & $r / \ldots$ & NE/IVA & N/MF. & بزركترين ريشه & \\
\hline
\end{tabular}

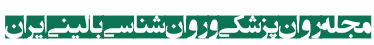

جدول F. تأثير درمان مبتنى بر تعهد و يذيرش (ACT) بر اهمال كارى تحصيلى و مؤلفهاى آن در دانشجويان

\begin{tabular}{|c|c|c|c|c|c|}
\hline سطح معنادارى & ضريب F & ميانغين مجذورات & ورجه آزادى & مجموع مجذورات & متغير هاي وابيته \\
\hline$+1++1$ & raVPaAT & $\operatorname{rgpr} / 1+1$ & 1 & FEPV/ +1 & اهمال كارى تحصيلى (در كل) \\
\hline $.1 .+1$ & $\Delta \Delta / \Lambda \cdot V$ & MFI/AqF & 1 & MFI/A9F & اهمال كارى در آمادكى براى امتحانات \\
\hline $.1 . .1$ & $M \cdot 1 \cdot 1$ & eqr/A9) & 1 & Eq4/A9) & اهمال كارى در انجام تكاليف درسى \\
\hline $.1 \%+1$ & AA/RTE & Drf/gt. & 1 & $\Delta F E / g F$. & اهمال كارى در مقالات و سمينارهاى بايانترم \\
\hline
\end{tabular}

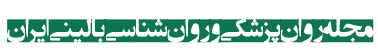

درمان در كاهش عواملى جون استرس، افسردكى، اضطراب،

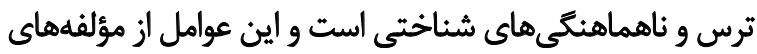

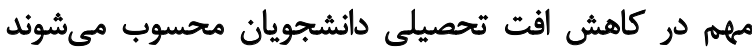

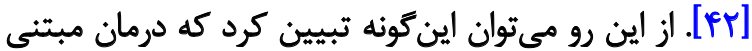

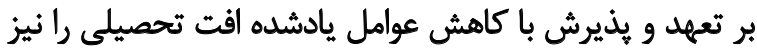

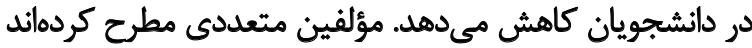

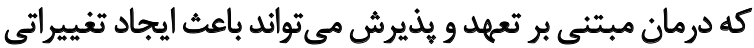

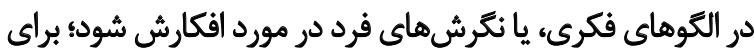

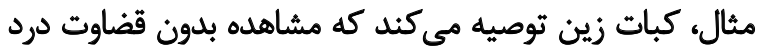

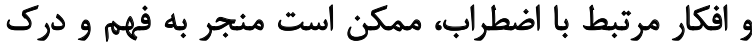

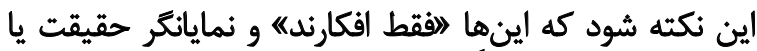

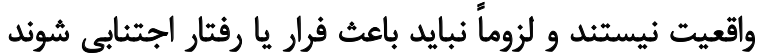

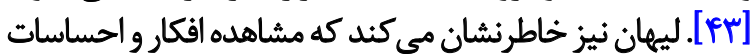

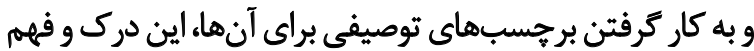

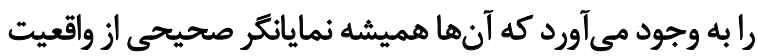

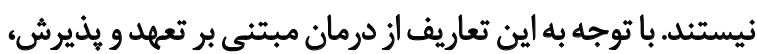

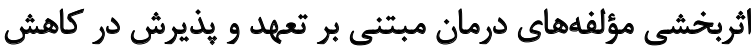

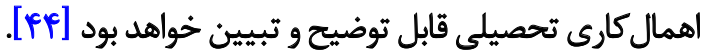

$$
\text { نتيجهئميرى }
$$

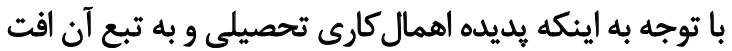

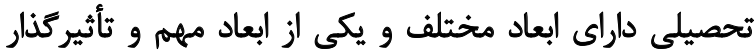

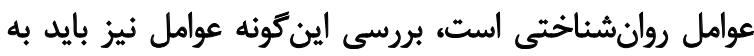

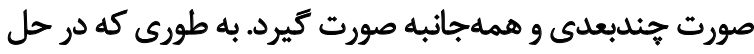

دانشجو به كار كرفته مىشود و دانشجو سعى دارد تا با به تعويق

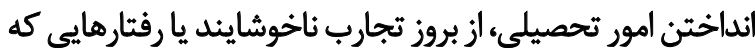

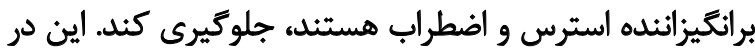

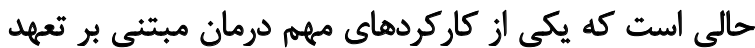

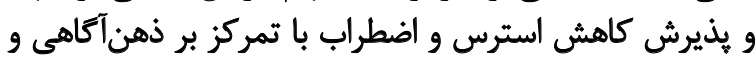

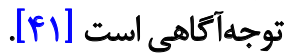

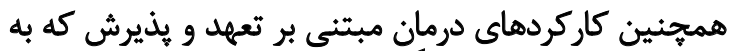

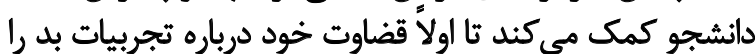

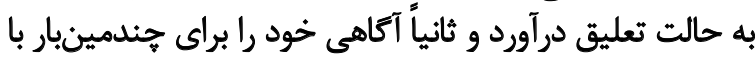

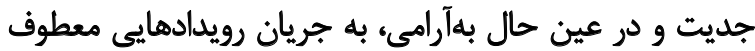

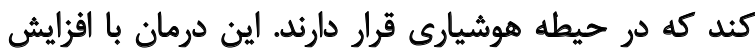

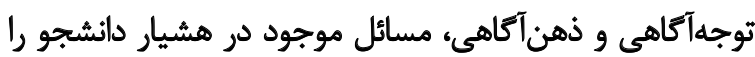

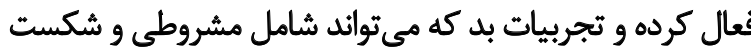

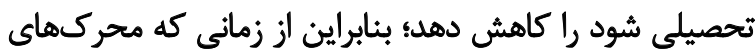

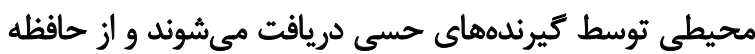

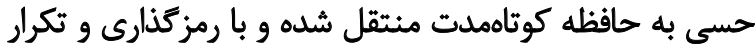

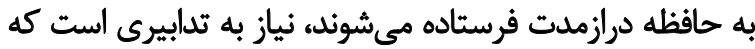

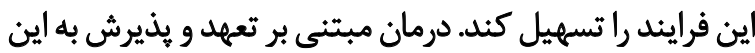

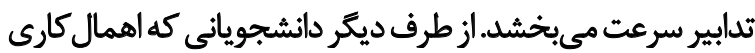

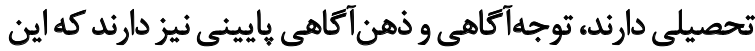

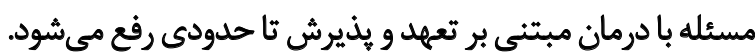
از طرف ديكر نتايج مطالعاتى كه در زميئه اثربخشى درمان

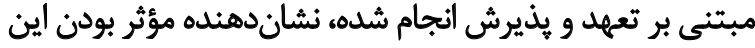




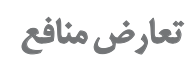

بنابر اظهار نويسندكان، اين مقاله تعارض منافع ندارد.

$$
\text { تشكر و قثدرواني }
$$

نويسندكان اين مقاله از مديريت و همكاران محترم مركز

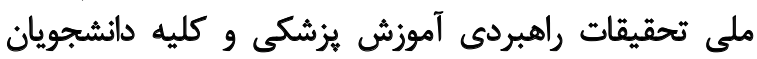

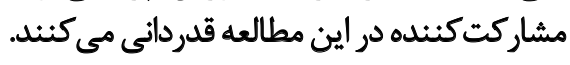

مسائل دانشجويان دهار افت تحصيلى در كنار حوزه آموزش،

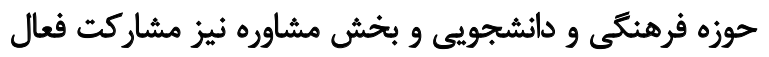

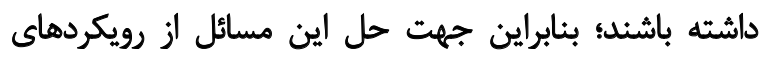

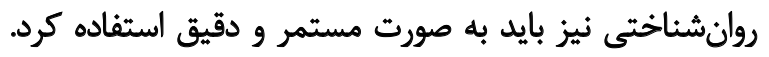

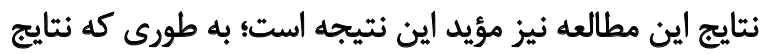

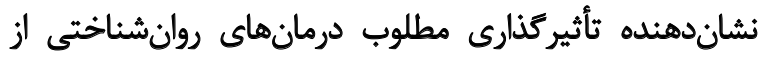

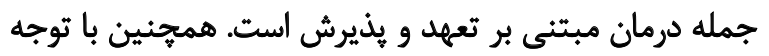

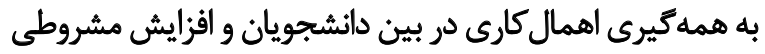

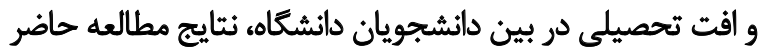

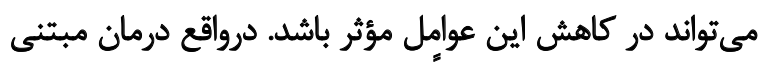

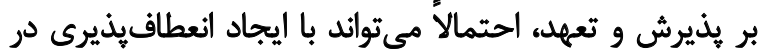

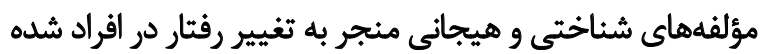

$$
\text { ودرنتيجه اثربخش باشد. }
$$

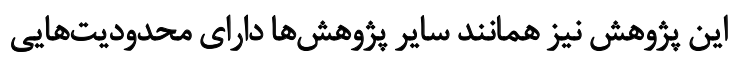

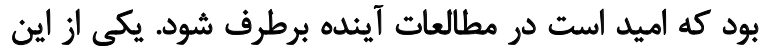

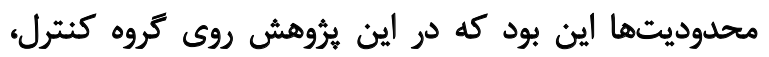

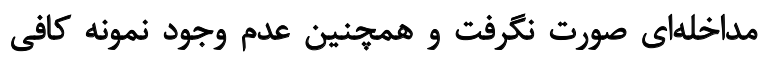

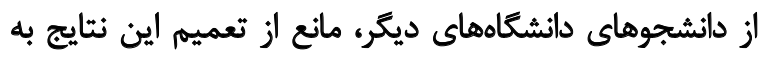

$$
\text { دانشجويان دانشعاههاى ديكر مى دانشود. }
$$

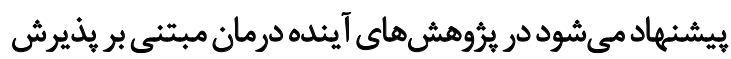

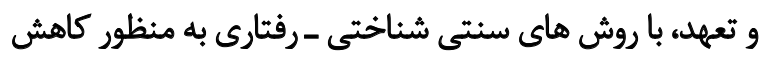

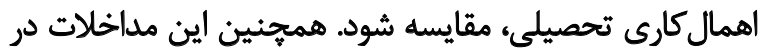
نمونه دانشآموزان نيز اجراشي، مقايشه شود.

مالاحظات اخلاقي

\section{بيروى أز اصول اخلاق يثوهش}

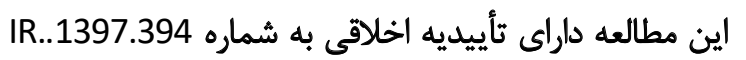

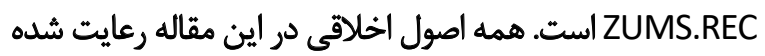

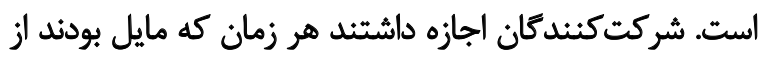

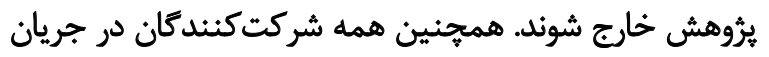

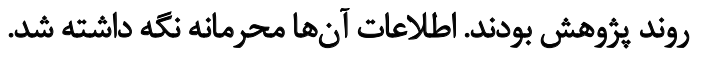

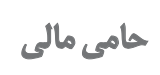

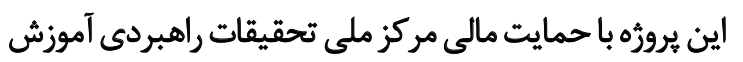

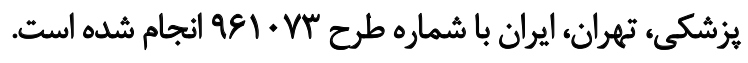

$$
\text { مشاركت نويسندكان }
$$

مفهومسازى: احسان فكور و عليرضا آرمانى كيان؛ تحقيق و

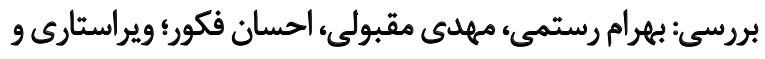

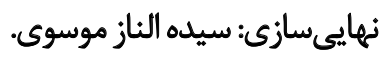




\section{References}

[1] Rasouli F, Sangani AR, Jangi P. [The relationship between academic procrastination, locus of control and achievement motivation with academic achievement in nursing student (Persian)]. Journal of Nursing Education. 2019; 8(1):21-8. http://jne.ir/ article-1-1026-en.html

[2] Bekleyen N. Understanding the academic procrastination attitude of language learners in Turkish universities. Educational Research and Reviews. 2017; 12(3):108-15. [DOI:10.5897/ ERR2016.3122]

[3] Shahbazian A, Bahadori Khosroshahi J. [Discrimination between normal and educational procrastination students based on Internet addiction (Persian)]. Daneshvar Medicine. 2017; 25(4):110. http://daneshvarmed.shahed.ac.ir/article_1796.html

[4] Balkis M, Duru E. Prevalence of academic procrastination behavior among pre-service teachers, and its relationship with demographics and individual preferences. Journal of Theory \& Practice in Education. 2009; 5(1):18-32. http:/ / citeseerx.ist.psu.edu/view$\mathrm{doc} /$ download?doi=10.1.1.824.8098\&rep=rep1\&type=pdf

[5] Tingting D, Xiulan Y, Xue G. Different procrastination types of college students: From a self-regulated learning perspective. Education Research Monthly. 2015; 10:014. https://en.cnki. com.cn/Article_en/CJFDTotal-YANG201510014.htm

[6] Asif SE. Clinical implication of academic procrastination. Alhambra, CA: Alliant International University; 2011. https:// books.google.com/books?id=WcKwMwEACAAJ\&dq

[7] Alexander ES, Onwuegbuzie AJ. Academic procrastination and the role of hope as a coping strategy. Personality and Individual Differences. 2007; 42(7):1301-10. [DOI:10.1016/j.paid.2006.10.008]

[8] Kim KR, Seo EH. The relationship between procrastination and academic performance: A meta-analysis. Personality and Individual Differences. 2015; 82:26-33. [DOI:10.1016/j. paid.2015.02.038]

[9] Motie H, Heidari M, Bagherian F, Zarani F. [Providing mindfulness-based educational package for evaluating academic procrastination (Persian)]. Iranian Journal of Psychiatry \& Clinical Psychology. 2019; 25(1):26-41. [DOI:10.32598/ijpcp.25.1.25]

[10] Ferrari JR. Still procrastinating: The no regrets guide to getting it done. Hoboken: Wiley; 2010. https:/ / books.google.com/ books?id=L4BDQxrME0IC\&dq

[11] Grunschel C, Patrzek J, Fries S. Exploring reasons and consequences of academic procrastination: An interview study. European Journal of Psychology of Education. 2013; 28(3):841-61. [DOI:10.1007/s10212-012-0143-4]

[12] Foroutan Bagha P, Kavousian J, Pasha Sharifi H. [The efficiency of procrastination reduction training on lowering self- handicapping and enhancing academic engagement among university students (Persian)]. Middle Eastern Journal of Disability Studies. 2018; 8:23. http://jdisabilstud.org/article-1-1133-en.html

[13] Rozental A, Forsström D, Nilsson S, Rizzo A, Carlbring P. Group versus Internet-based cognitive-behavioral therapy for procrastination: Study protocol for a randomized controlled trial. Internet Interventions. 2014; 1(2):84-9. [DOI:10.1016/j.invent.2014.05.005]

[14] Rozental A, Forsström D, Lindner P, Nilsson S, Mårtensson L, Rizzo A, et al. Treating procrastination using cogni- tive behavior therapy: A pragmatic randomized controlled trial comparing treatment delivered via the internet or in groups. Behavior Therapy. 2018; 49(2):180-97. [DOI:10.1016/j. beth.2017.08.002] [PMID]

[15] Talask G, de Carvalho MR. Cognitive behavioral based treatment for procrastination. Psychology and Behavioral Science International Journal. 2017; 8(1):555727. [DOI:10.19080/PBSIJ.2017.08.555727]

[16] Kobori O, Dighton G, Hunter R. Does perfectionism impact adherence to homework assignment? A preliminary pilot study of perfectionism and procrastination of CBT homework. Behavioural and Cognitive Psychotherapy. 2020; 48(2):243-7. [DOI:10.1017/S1352465819000547] [PMID]

[17] Ellis A, \& Knaus WJ. Overcoming procrastination: Or how to Think and Act Rationally in Spite of Life's Inevitable Hassles, New York: Institute for Rational Living. 1979. https:/ /books. google.com/books?id=bpn0JgAACAAJ\&dq

[18] Rozental A, Bennett S, Forsström D, Ebert DD, Shafran R Andersson G, et al. Targeting procrastination using psychological treatments: A systematic review and meta-analysis. Frontiers in Psychology. 2018; 9:1588. https://doi.org/10.3389/ fpsyg.2018.01588

[19] van Eerde W, Klingsieck KB. Overcoming procrastination? A meta-analysis of intervention studies. Educational Research Review. 2018; 25:73-85. [DOI:10.1016/j.edurev.2018.09.002]

[20] Eckert M, Ebert DD, Lehr D, Sieland B, Berking M. Overcome procrastination: Enhancing emotion regulation skills reduce procrastination. Learning and Individual Differences. 2016; 52:10-8. [DOI:10.1016/j.lindif.2016.10.001]

[21] Hayes SC, Luoma JB, Bond FW, Masuda A, Lillis J. Acceptance and commitment therapy: Model, processes and outcomes. Behaviour Research and Therapy. 2006; 44(1):1-25. [DOI:10.1016/j.brat.2005.06.006] [PMID]

[22] Dionne F. Using acceptance and mindfulness to reduce procrastination among university students: Results from a pilot study. Revista Prâksis. 2016; 1(13):8-20. [DOI:10.25112/ rp.v1i0.431]

[23] Hayes SC, Levin ME, Plumb-Vilardaga J, Villatte JL, Pistorello J. Acceptance and commitment therapy and contextual behavioral science: Examining the progress of a distinctive model of behavioral and cognitive therapy. Behavior Therapy 2013; 44(2):180-98. [DOI:10.1016/j.beth.2009.08.002] [PMID] [PMCID]

[24] Flaxman PE, Bond FW. A randomized worksite comparison of acceptance and commitment therapy and stress inoculation training. Behavior Research and Therapy. 2010; 48(8):816-20. [DOI:10.1016/j.brat.2010.05.004] [PMID]

[25] Bluett EJ, Homan KJ, Morrison KL, Levin ME, Twohig MP. Acceptance and commitment therapy for anxiety and OCD spectrum disorders: An empirical review. Journal of Anxiety Disorders. 2014; 28(6):612-24. [DOI:10.1016/j. janxdis.2014.06.008] [PMID]

[26] Yadavaia JE, Hayes SC, Vilardaga R. Using acceptance and commitment therapy to increase self-compassion: A randomized controlled trial. Journal of Contextual Behavioral Science. 2014; 3(4):248-57. [DOI:10.1016/j.jcbs.2014.09.002] [PMID] [PMCID] 
[27] Hoffmann D, Halsboe L, Eilenberg T, Jensen JS, Frostholm L. A pilot study of processes of change in group-based acceptance and commitment therapy for health anxiety. Journal of Contextual Behavioral Science. 2014; 3(3):189-95. [DOI:10.1016/j. jcbs.2014.04.004]

[28] Fledderus M, Bohlmeijer ET, Fox JP, Schreurs KMG, Spinhoven P. The role of psychological flexibility in a self-help acceptance and commitment therapy intervention for psychological distress in a randomized controlled trial. Behavior Research and Therapy. 2013; 51(3):142-51. [DOI:10.1016/j.brat.2012.11.007] [PMID]

[29] Eskandari M, Rostami A, Hojati A, Kolifarhood G. [Trends of academic failure and its demographic determinants among medical students in Zanjan University of Medical Sciences (Persian)]. Iranian Journal of Medical Education. 2014; 14(2):174-83. http:// ijme.mui.ac.ir/article-1-2955-en.html

[30] Kiani Q, Mahdion H. [The relationship between cognitive-emotional regulation and study skills with academic procrastination in students of the faculty of health and paramedicine, Zanjan University of Medical Sciences (Persian)]. Journal of Medical Education Development. 2017; 10(26):72-82. [DOI:10.29252/edcj.10.26.72]

[31] Safavi SZ, Rostami B, Moein Z, Rostami H, Fakour E. [Factors Affecting the Academic Achievement of pharmacy school s students in Zanjan University of Medical Sciences (Persian)]. Journal of Medical Education Development. 2017; 10(25):51-8. http:// zums.ac.ir/edujournal/article-1-737-en.html

[32] Gagnon J, Pychyl TA. Committed action: An initial study on its association to procrastination in academic settings. Journal of Contextual Behavioral Science. 2016; 5(2):97-102. [DOI:10.1016/j. jcbs.2016.04.002]

[33] Scent CL, Boes SR. Acceptance and commitment training: A brief intervention to reduce procrastination among college students. Journal of College Student Psychotherapy. 2014; 28(2):14456. [DOI:10.1080/87568225.2014.883887]

[34] Wang Sh, Zhou Y, Yu Sh, Ran LW, Liu XP, Chen YF. Acceptance and commitment therapy and cognitive-behavioral therapy as treatments for academic procrastination: A randomized controlled group session. Research on Social Work Practice. 2017; 27(1):48-58. [DOI:10.1177/1049731515577890]

[35] Glick DM, Millstein DJ, Orsillo SM. A preliminary investigation of the role of psychological inflexibility in academic procrastination. Journal of Contextual Behavioral Science. 2014; 3(2):81-8. [DOI:10.1016/j.jcbs.2014.04.002]

[36] Rezapour Mirsaleh Y, Ahmadi Ardakani Z, Shiri M. [Effectiveness of Acceptance and Commitment Therapy (ACT) on academic performance of veterans' children (Persian)]. Iranian Journal of War and Public Health. 2018; 10(1):33-9. [DOI:10.29252/ ijwph.10.1.33]

[37] Pourabdol S, Sobhigharamaleki N, Bastami M, Ghazanfari H. [The effect of acceptance and commitment therapy in decreasing academic procrastination among students with Specific Learning Disorder (SLD) (Persian)]. Biquarterly Journal of Cognitive Strategies in Learning. 2016; 4(6):157-70. [DOI:10.22084/J.PSYCHOGY.2016.1466]

[38] Golmahammadian M, Rashidi AR, Parvaneh A. [Effectiveness of training based on acceptance and commitment on cognitive emotion regulation and beliefs efficacy in the female students (Persian)]. Educational Psychology. 2018; 14(47):1-23. [DOI:10.22054/ JEP.2018.22533.1831]
[39] Heshmati A, Saed O, Mohammadi Baytamar J, Zenoozian S, Yousefi F. [The efficacy of group acceptance and commitment therapy on reducing academic procrastination and improving difficulty in emotion regulation: A randomized clinical trial (Persian)]. Scientific Journal of Kurdistan University of Medical Sciences. 2018; 23(5);65-77. http:/ / sjku.muk.ac.ir/article-1-4316-en.html

[40] Hadadranjbar S, Sadipour E, Dortaj F, Delavar A, Ebrahimi Qavam S. [Effect of education based on acceptance and commitment approach in reducing students' self -defeating behaviors: According to the educational package developed (Persian)]. Research in Medical Education. 2018; 10(2):8-17. [DOI:10.29252/rme.10.2.8]

[41] Grunschel C, Schwinger M, Steinmayr R, Fries S. Effects of using motivational regulation strategies on students' academic procrastination, academic performance, and well-being. Learning and Individual Differences. 2016; 49:162-70. [DOI:10.1016/j. lindif.2016.06.008]

[42] Harris R. ACT made simple: An easy-to-read primer on acceptance and commitment therapy. Oakland, CA: New Harbinger Publications; 2009. https://books.google.com/ books?id=h7g9kc_cfYMC\&dq

[43] Hayes SC, Strosahl KD. A practical guide to acceptance and commitment therapy. Boston, MA: Springer; 2004. [DOI:10.1007/978-0-387-23369-7]

[44] Steel P, Klingsieck KB. Academic procrastination: Psychological antecedents revisited. Australian Psychologist. 2016; 51(1):36-46. [DOI:10.1111/ap.12173] 